\title{
PSEUDO-INDEX OF FANO MANIFOLDS AND SMOOTH BLOW-UPS
}

\author{
LAURENT BONAVERO
}

\begin{abstract}
Suppose $\pi: X \rightarrow Y$ is a smooth blow-up along a submanifold $Z$ of $Y$ between complex Fano manifolds $X$ and $Y$ of pseudo-indices $i_{X}$ and $i_{Y}$ respectively (recall that $i_{X}$ is defined by $i_{X}:=$ $\min \left\{-K_{X} \cdot C \mid C\right.$ is a rational curve of $\left.\left.X\right\}\right)$. We prove that $i_{X} \leq i_{Y}$ if $2 \operatorname{dim}(Z)<\operatorname{dim}(Y)+i_{Y}-1$ and show that this result is optimal by classifying the "boundary" cases. As expected, these results are obtained by studying rational curves on $X$ and $Y$.
\end{abstract}

\section{Statement of the Results}

1.1. Introduction. When studying surjective morphisms $f: X \rightarrow Y$ between smooth Fano manifolds $X$ and $Y$ of the same dimension, one generally observes that the anticanonical bundle $-K_{Y}$ of $Y$ is "more positive" than the anti-canonical bundle $-K_{X}$ of $X$, one of the most important results in this direction being the famous theorem of Lazarsfeld La83] stating that if $\mathbb{P}^{n} \rightarrow Y$ is a surjective morphism from $\mathbb{P}^{n}$ to an $n$-dimensional manifold $Y$, then $Y \simeq \mathbb{P}^{n}$.

For a Fano manifold $X$ (i.e., a complex manifold with ample anti-canonical line bundle $-K_{X}$ ), one defines two integers called the index $r_{X}$ and the pseudo-index $i_{X}$ of $X$ by

$$
r_{X}:=\max \left\{m \in \mathbb{N} \mid-K_{X}=m L \text { with } L \in \operatorname{Pic}(X)\right\}
$$

and

$$
i_{X}:=\min \left\{-K_{X} \cdot C \mid C \text { is a rational curve of } X\right\} .
$$

Of course, $i_{X}$ is a multiple of $r_{X}$ and many results are known for these numbers. Among others, Fano manifolds of dimension $n$ with large index (namely bigger than $n-2$ ) are classified (see [IP99] for a complete survey on Fano manifolds), the situation being much more complicated for the pseudo-index: one knows that $i_{X} \leq n+1$ by Mori theory, equality holding if and only if $X \simeq \mathbb{P}^{n}$ CMS00.

1.2. The main result. Let us start with an easy remark: let $Y$ be a complex manifold of dimension $n$, let $Z$ be a connected submanifold of $Y$, let $X:=B_{Z}(Y)$ be the blow-up of $Y$ with center $Z$ and let $E$ be the exceptional divisor of $\pi: X \rightarrow Y$. We classically have

$$
H^{2}(X, \mathbb{Z}) \simeq H^{2}(Y, \mathbb{Z}) \oplus \mathbb{Z} \cdot E \text { and } K_{X}=\pi^{*} K_{Y}+(n-\operatorname{dim}(Z)-1) E .
$$

Therefore, if both $Y$ and $X$ are Fano, $r_{X}$ is equal to the greatest common divisor of $r_{Y}$ and $n-\operatorname{dim}(Z)-1$, which implies in particular that $r_{X} \leq r_{Y}$ and confirms the philosophy described above.

In this Note, we study the behaviour of the pseudo-index with respect to smooth blowups. Quite surprisingly, this behaviour depends on the dimension of the center of the blow-up. Our precise results are the following.

Date: September 2003.

Key-words : Fano manifolds, smooth blow-up, rational curves. A.M.S. classification : 14J45, 14E30, 14E05. 
Theorem 1. Let $Y$ be a complex manifold of dimension n, let $Z$ be a connected submanifold of $Y$ and let $X:=B_{Z}(Y)$ be the blow-up of $Y$ with center $Z$. Suppose both $Y$ and $X$ are Fano.

(i) If $2 \operatorname{dim}(Z)<n+i_{Y}-1$, then $i_{X} \leq i_{Y}$,

(ii) if $2 \operatorname{dim}(Z)=n+i_{Y}-1$ and $i_{Y} \geq 2$, then $i_{X} \leq i_{Y}$,

(iii) if $\operatorname{dim}(Z)<n / 2$, then $i_{X} \leq i_{Y}$.

Of course, (iii) is an obvious consequence of (i) since $i_{Y} \geq 1$. This result says that the pseudo-index has the "expected behaviour" when the center of the blow-up has small dimension. Remark that the case where $\operatorname{dim}(Z)=0$ could be proved by looking at the classification given in BCW02 and the case where $\operatorname{dim}(Z)=1$ is Proposition 3.7 of BCDD03.

Let us now give an example where $i_{X}$ is bigger than $i_{Y}$. In the following proposition (as in the whole paper), we do not follow Grothendieck's convention: $\mathbb{P}(V)$ denotes the projective space of lines of the vector space $V$.

Proposition 1. Let $n:=2 m$ be an even integer, let $\mathcal{E}$ be the following rank $m+1$ vector bundle over $\mathbb{P}^{m}$ :

$$
\mathcal{E}=\mathcal{O}_{\mathbb{P} m}^{\oplus m} \oplus \mathcal{O}_{\mathbb{P} m}(1)
$$

and let $Y_{n}$ be the $n$-dimensional manifold $Y_{n}=\mathbb{P}(\mathcal{E})$. The trivial rank $m$-subbundle of $\mathcal{E}$ defines a submanifold $Z_{m}$ isomorphic to $\mathbb{P}^{n / 2}$ with normal bundle $N_{Z_{m} / Y_{n}}$ isomorphic to $\mathcal{O}_{\mathbb{P}^{m}}(-1)^{\oplus m}$. Finally, let $\pi_{n}: X_{n}=B_{Z_{m}}\left(Y_{n}\right) \rightarrow Y_{n}$ be the blow-up of $Y_{n}$ along $Z_{m}$. Then $Y_{n}$ and $X_{n}$ are Fano manifolds of dimension $n$ if $n \geq 4$. Moreover $i_{Y_{n}}=1$, $i_{X_{4}}=1$ and $i_{X_{n}}=2$ if $n \geq 6$.

Therefore, the inequalities of Theorem 1 are optimal: for any $n=2 m \geq 6, \pi_{n}: X_{n}=$ $B_{Z_{m}}\left(Y_{n}\right) \rightarrow Y_{n}$ is a blow-up with smooth connected center between Fano manifolds with $\operatorname{dim}\left(Z_{m}\right)=\operatorname{dim}\left(X_{n}\right) / 2$ and $i_{X_{n}}>i_{Y_{n}}$.

Proof of Proposition 1. Since $X_{n}$ and $Y_{n}$ are naturally toric manifolds, it is enough to compute the anti-canonical degree of invariant (rational) curves. If $d$ is a line contained in $Z_{m}$, then $-K_{Y_{n}} \cdot d=1$, which gives $i_{Y_{n}}=1$. The Fano manifold $X_{n}$ is isomorphic to the $\mathbb{P}^{1}$-bundle $\mathbb{P}_{\mathbb{P}^{m-1} \times \mathbb{P}^{m}}\left(\mathcal{O}_{\mathbb{P}^{m-1} \times \mathbb{P}^{m}} \oplus \mathcal{O}_{\mathbb{P}^{m-1} \times \mathbb{P}^{m}}(1,1)\right)$ over $\mathbb{P}^{m-1} \times \mathbb{P}^{m}$ hence $i_{X_{n}} \leq 2$ (the $\mathbb{P}^{1}$-fibers having anti-canonical degree equal to 2). Let $E \simeq \mathbb{P}^{m-1} \times \mathbb{P}^{m}$ be the exceptional divisor of $\pi_{n}$ : the lines contained in a $\mathbb{P}^{m-1} \times\{*\} \subset E$ have anti-canonical degree equal to $m-1$, hence $i_{X_{4}}=1$ and $i_{X_{n}}=2$ if $n \geq 6$.

Remark: for $n \geq 8$, the previous computations show that the rational curves in $X_{n}$ of minimal anti-canonical degree are not mapped by $\pi_{n}$ to curves of minimal anti-canonical degree in $Y_{n}$.

Let us now discuss in more details the optimality of Theorem 1 by classifying the "boundary cases".

Theorem 2. Let $\pi: X \rightarrow Y$ be a blow-up with smooth connected center $Z$ between Fano manifolds $X$ and $Y$ of dimension $n$. If $2 \operatorname{dim}(Z)=n+i_{Y}-1$ then $i_{X} \leq i_{Y}$ unless $n \geq 6$ is even, $X=X_{n}, Y=Y_{n}$ and $\pi=\pi_{n}$.

1.3. Some consequences. The results above have the following consequences when the pseudo-index of $Y$ is large or in low dimensions.

Corollary 1. Let $\pi: X \rightarrow Y$ be a blow-up with smooth connected center between Fano manifolds $X$ and $Y$ of dimension $n$. 
(i) If $i_{Y}>n / 3-1$, then $i_{X} \leq i_{Y}$.

(ii) If $i_{Y}=n / 3-1$, then $i_{X} \leq i_{Y}$ unless $n=6, X=X_{6}, Y=Y_{6}$ and $\pi=\pi_{6}$.

Proof of Corollary 1 .

Proof of (i). Suppose by contradiction that $i_{X}>i_{Y}$. Then by Theorem प(i), $2 \operatorname{dim}(Z) \geq$ $n+i_{Y}-1$. But the lines contained in the non-trivial fibers of the blow-up are rational curves of anti-canonical degree $n-1-\operatorname{dim}(Z)$, therefore $n-1-\operatorname{dim}(Z) \geq i_{X}>i_{Y}$, hence

$$
n-1-i_{Y} \geq \operatorname{dim}(Z)+1 \geq n / 2+i_{Y} / 2+1 / 2
$$

and $i_{Y} \leq n / 3-1$, a contradiction.

Proof of (ii). Suppose that $i_{Y}=n / 3-1$ and that $i_{X}>i_{Y}$. The previous computations implies that every inequality occuring in the proof of (i) is an equality. In particular, one has $2 \operatorname{dim}(Z)=n+i_{Y}-1$. Therefore, Theorem 2 implies that $n$ is even and $Y=Y_{n}$. In particular, $i_{Y}=1=n / 3-1$, hence $n=6$, which ends the proof.

Remark that according to the generalised Mukai conjecture, as stated and studied in BCDD03, Fano manifolds $Y$ of dimension $n \geq 6$ with $i_{Y}>n / 3-1$ should have Picard number $\rho_{Y}$ satisfying $\rho_{Y}<\frac{3 n}{n-6}$. Corollary 1 has the immediate following corollary.

Corollary 2. Let $\pi: X \rightarrow Y$ be a blow-up with smooth connected center between Fano manifolds $X$ and $Y$ of dimension $n$.

(i) If $n \leq 5$, then $i_{X} \leq i_{Y}$.

(ii) If $n=6$, then $i_{X} \leq i_{Y}$ unless $X=X_{6}, Y=Y_{6}$ and $\pi=\pi_{6}$.

\section{Proofs}

2.1. Proof of Theorem 1, It is enough to prove assertion (i), since (iii) is an obvious consequence of (i) and (ii) is an immediate consequence of Theorem 2 (note that the Fano manifolds $Y_{n}$ have pseudo-index 1).

Let $\pi: X=B_{Z}(Y) \rightarrow Y$ be a blow-up with smooth center $Z$ between Fano manifolds $X$ and $Y$. We will denote by $E=\pi^{-1}(Z)$ the exceptional divisor of $\pi$. The basic idea is very simple: we take a rational curve $C$ in $Y$ such that $-K_{Y} \cdot C=i_{Y}$ and we want to show that there is a rational curve $\tilde{C}$ in $X$, mapping surjectively to $C$ by $\pi$, such that $-K_{X} \cdot \tilde{C} \leq i_{Y}$.

Suppose first that there is a rational curve $C$ in $Y$ such that $-K_{Y} \cdot C=i_{Y}$ and such that $C$ is not contained in $Z$. The strict transform $\tilde{C}$ of $C$ is a rational curve satisfying $E \cdot \tilde{C} \geq 0$ and the formula $-K_{X}=\pi^{*}\left(-K_{Y}\right)-(n-1-\operatorname{dim}(Z)) E$ immediately implies that $-K_{X} \cdot \tilde{C} \leq i_{Y}$.

Now take a rational curve $C$ in $Y$ such that $-K_{Y} \cdot C=i_{Y}$ and assume $C \subset Z$. Let us decompose $N_{Z / Y \mid C}$ (we allow here a slight abuse of notations, since $C$ might be a singular rational curve, we should rather write $\nu^{*}\left(N_{Z / Y \mid C}\right)$ where $\nu: \mathbb{P}^{1} \rightarrow C$ is the normalisation of $C$ ):

$$
N_{Z / Y \mid C}=\bigoplus_{i=1}^{r} \mathcal{O}_{\mathbb{P}^{1}}\left(a_{i}\right)
$$

where $r=n-\operatorname{dim}(Z)$. The sub-line bundles $\mathcal{O}_{\mathbb{P}^{1}}\left(a_{i}\right)$ of $N_{Y / Z \mid C}$ define rational curves $\tilde{C}_{i}$ of $E$ satisfying $-K_{X} \cdot \tilde{C}_{i}=-K_{Y} \cdot C-(r-1) a_{i}$. Therefore, we are done if there exists $i$ such that $a_{i} \geq 0$. Suppose the contrary, namely that $a_{i} \leq-1$ for all $i$. Then

$$
-K_{Z} \cdot C=-K_{Y} \cdot C-\operatorname{deg}\left(N_{Z / Y \mid C}\right) \geq i_{Y}+\operatorname{rk}\left(N_{Z / Y \mid C}\right)=i_{Y}+n-\operatorname{dim}(Z) .
$$


Under the assumption $2 \operatorname{dim}(Z)<n+i_{Y}-1$, we get $-K_{Z} \cdot C>\operatorname{dim}(Z)+1$ hence, by Mori's bend-and-break lemma (see for example Deb01, p. 58), the curve $C$ is numerically equivalent in $Z$, hence in $Y$, to a connected nonintegral effective rational 1-cycle (passing through 2 arbitrary fixed points of $C$ ). Each reduced irreducible component of this 1-cycle has $-K_{Y}$ anti-canonical degree strictly less than $i_{Y}$, contradiction!

Remarks: in the above proof, we only used that $-K_{Y} \cdot C>0$ for any rational curve of $Y$. Moreover, the previous proof also shows the following. Let $Y$ be a complex manifold, let $Z$ be a connected submanifold of $Y$ and let $X:=B_{Z}(Y)$ be the blow-up of $Y$ with center $Z$. Suppose both $Y$ and $X$ are Fano and $i_{X}>i_{Y}$. Then any rational curve $C$ satisfying $-K_{Y} \cdot C=i_{Y}$ is contained in $Z$ and for any such curve $C$, the vector bundle $N_{Z / Y \mid C}^{*}$ is ample.

2.2. Proof of Theorem 2, This proof assumes that the reader has some familiarity with Mori theory, see for example [Deb01] for a nice introduction.

By the remark above, if $i_{X}>i_{Y}$, any rational curve $C$ in $Y$ such that $-K_{Y} \cdot C=i_{Y}$ is contained in $Z$. For such a curve $C$, which has minimal degree with respect to an ample line bundle, its deformations in $Z$ containing a given point cover a subvariety of dimension $\geq-K_{Z} \cdot C-1$ (recall that this an easy consequence of Riemann-Roch formula and the bend-and-break lemma, see for example Deb01, §6.5). Since the computations in the proof of Theorem 1(i) show that $-K_{Z} \cdot C=\operatorname{dim}(Z)+1$, the deformations of $C$ in $Z$ containing a given point cover $Z$. Therefore, the Picard number of $Z$ is one (see Ko96, IV 3.13.3). One deduces that any rational curve $C^{\prime}$ of $Z$ is numerically proportional (in $\mathrm{N}_{1}(Z)$ ) to $C$, and since $C$ has minimal anti-canonical degree in $Y$, $C^{\prime}$ satisfies $-K_{Z} \cdot C^{\prime} \geq \operatorname{dim}(Z)+1$, hence $Z \simeq \mathbb{P}^{\operatorname{dim}(Z)}$ by CMS00. Finally, the computations above also show that for any line $d$ in $Z, N_{Z / Y \mid d} \simeq \mathcal{O}_{d}(-1)^{\oplus n-\operatorname{dim}(Z)}$,

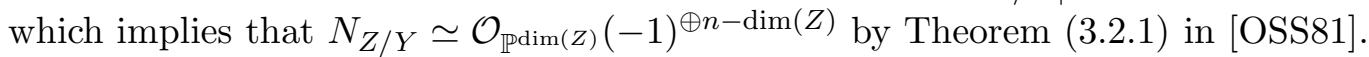

Let $E=\mathbb{P}^{\operatorname{dim}(Z)} \times \mathbb{P}^{n-\operatorname{dim}(Z)-1}$ be the exceptional divisor of $\pi$, and let $\omega$ be a Mori extremal rational curve in $X$ such that $E \cdot \omega>0$ (such a curve exists by the classical following argument: take any curve with strictly positive intersection with $E$ and decompose it in the Mori cone $\mathrm{NE}(X)$ as an effective combination of extremal curves, at least one of these curves has strictly positive intersection with $E$ ). The corresponding Mori contraction $\varphi_{\omega}$ satisfies Wiśniewski's inequality Wi91:

$$
\operatorname{dim}\left(\operatorname{Exc}\left(\varphi_{\omega}\right)\right)+\operatorname{dim}(f) \geq n-1+i_{X}
$$

where $\operatorname{Exc}\left(\varphi_{\omega}\right)$ is the locus of contracted curves, and $f$ is any non-trivial fiber of $\varphi_{\omega}$. Since every contracted curve is proportional to $\omega$ in $\mathrm{N}_{1}(X)$ and since $\mathcal{O}(E)_{\mid E} \simeq$ $\mathcal{O}_{\mathbb{P}^{\operatorname{dim}}(Z)} \times \mathbb{P}^{n-\operatorname{dim}(Z)-1}(-1,-1)$, none of these curves are contained in $E$, therefore any non-trivial fiber $f$ of $\varphi_{\omega}$ satisfies $\operatorname{dim}(f)=1$. Moreover, $i_{X} \geq 2$, therefore $\operatorname{Exc}\left(\varphi_{\omega}\right)=X$ by Wiśniewski's inequality above and $\varphi_{\omega}$ is a fibration which, by Ando's classification An85, is a smooth $\mathbb{P}^{1}$-bundle over an $(n-1)$-dimensional Fano manifold $X^{\prime}$. Moreover, $i_{X}=2$, hence $i_{Y}=1$, therefore $\operatorname{dim}(Z)=n / 2$.

Let us show now that $E \cdot f=1$ for any fiber of $\varphi_{\omega}$. Indeed, if $\mathbb{P}^{1} \rightarrow X^{\prime}$ is a rational curve of $X^{\prime}$, the surface $S=\mathbb{P}^{1} \times_{X^{\prime}} X$ is a ruled surface, i.e., a Hirzebruch surface, and the exceptional curve of $S$ is nothing else than $\mathbb{P}^{1} \times_{X^{\prime}} E$, which is a section of $S \rightarrow \mathbb{P}^{1}$. Hence $E \cdot f=1$ for any fiber of $\varphi_{\omega}$.

One immediately deduces that $\varphi_{\omega}: E \rightarrow X^{\prime}$ is an isomorphism, hence $X=\mathbb{P}(\mathcal{E})$ for some rank 2 bundle $\mathcal{E}$ over $X^{\prime} \simeq E \simeq \mathbb{P}^{n / 2} \times \mathbb{P}^{n / 2-1}$, and $E$ defines a sub-line bundle 
of $\mathcal{E}$. Therefore $\mathcal{E}$ splits and since $N_{E / X} \simeq \mathcal{O}_{\mathbb{P}^{n / 2} \times \mathbb{P}^{n / 2-1}}(-1,-1)$, one deduces that

$$
X \simeq \mathbb{P}\left(\mathcal{O}_{\mathbb{P}^{n / 2} \times \mathbb{P}^{n / 2-1}} \oplus \mathcal{O}_{\mathbb{P}^{n / 2} \times \mathbb{P}^{n / 2-1}}(1,1)\right),
$$

which ends the proof.

\section{Some COMments AND SOME MORE EXAMPLES}

3.1. On the normal bundle of the center. The following proposition sheds some light on the example explained in Proposition 1.

Proposition 2. Let $\pi: X \rightarrow Y$ be a blow-up with smooth connected center $Z$ between Fano manifolds $X$ and $Y$ of dimension n. Suppose moreover that the conormal bundle $N_{Z / Y}^{*}$ is ample. Then,

(i) either $i_{X} \leq i_{Y}$,

(ii) or $i_{X}=2, i_{Y}=1, Z$ is a Fano manifold, $X$ is a $\mathbb{P}^{1}$-bundle over the $(n-1)$ dimensional Fano manifold $\mathbb{P}\left(N_{Z / Y}\right)$ and $Y$ is a $\mathbb{P}^{n-\operatorname{dim}(Z)}$-bundle over $Z$.

(Sketch of) proof. Suppose $i_{X}>i_{Y}$ and let denote by $E$ the exceptional divisor of $\pi$. Since $N_{Z / Y}^{*}$ is ample, $-E_{\mid E}$ is also ample and by Grauert's criterion, $E$ is contractible to a point. Moreover, if $\omega$ is a Mori extremal rational curve in $X$ such that $E \cdot \omega>0$, using the same arguments as in the proof of Theorem 2 the corresponding Mori contraction $\varphi_{\omega}$ is a $\mathbb{P}^{1}$-bundle over the Fano manifold $E \simeq \mathbb{P}\left(N_{Z / Y}\right)$. Finally, $Z$ is Fano by [SW90] and one has $\rho_{Y}+1=\rho_{X}=\rho_{E}+1=\rho_{Z}+2$, hence $\rho_{Y}=\rho_{Z}+1$. This implies that there is at least one Mori extremal curve of $Y$ which is not contained in $Z$. Since $\pi$ is surjective, the Mori cone $\mathrm{NE}(Y)$ is generated by the images of Mori extremal curve of $X$, which are contained in $E$, except for the fibers $f$ of the $\mathbb{P}^{1}$-bundle structure $X \rightarrow E$. This implies that $\pi(f)$ is extremal in $Y$ and the corresponding extremal contraction $\psi: Y \rightarrow W$ is a fibration. But then, the fibers of $\psi$ have dimension less or equal to $n-\operatorname{dim}(Z)$, hence equal to $n-\operatorname{dim}(Z)$ since $-K_{Y} \cdot \pi(f)=n-\operatorname{dim}(Z)+1$. Thereore the generic fiber of $\psi$ is $\mathbb{P}^{n-\operatorname{dim}(Z)}$, and finally every fiber of $\psi$ is $\mathbb{P}^{n-\operatorname{dim}(Z)}$ and meets $Z$ transversally at exactly one point (all this is verified since on $X$, the extremal contraction associated to $f$ is a $\mathbb{P}^{1}$-bundle). Finally, $W \simeq Z$.

3.2. Some examples. The previous Proposition implies that if $\pi: X \rightarrow Y$ is a blowup with smooth connected center $Z$ between Fano manifolds $X$ and $Y$ with $i_{X}>i_{Y} \geq 2$, then the conormal bundle $N_{Z / Y}^{*}$ is not ample, althought its restriction to any rational curve of minimal $-K_{Y}$-degree (recall that such a curve has to be contained in $Z$ ) is ample as we saw in the proof of Theorem 11! It is therefore the good place to give a list of examples (communicated to me by Cinzia Casagrande) of blow-ups $\pi: X \rightarrow Y$ with smooth connected center $Z$ between Fano manifolds $X$ and $Y$ with $i_{X}>i_{Y} \geq 2$.

Examples. Let $a, d, r$ and $s$ be positive integers, let $\mathcal{E}$ be the following rank $r+s$ vector bundle over $\mathbb{P}^{a}: \mathcal{E}=\mathcal{O}_{\mathbb{P} a}^{\oplus r} \oplus \mathcal{O}_{\mathbb{P}^{a}}(d)^{\oplus s}$ and let $\mathcal{F}=\mathcal{O}_{\mathbb{P}^{a}}(d)^{\oplus s}$ be the rank $s$ vector subbundle of $\mathcal{E}$ defined by the $\mathcal{O}_{\mathbb{P a}}(d)$ 's factors. Define $Y:=\mathbb{P}(\mathcal{E})$ and $Z:=\mathbb{P}(\mathcal{F}) \simeq$ $\mathbb{P}^{a} \times \mathbb{P}^{s-1}$. This submanifold $Z$ of $Y$ has codimension $r$ and normal bundle $N_{Z / Y}$ equal to $\mathcal{O}_{\mathbb{P}^{a} \times \mathbb{P}^{s-1}}(-d, 1)^{\oplus r}$. Finally, let $X:=B_{Z}(Y)$ be the blow-up of $Y$ with center $Z$. Easy computations show that $Y$ is Fano if and only if $a \geq r d$ and $X$ is Fano if and only if $a \geq d$, and that under these assumptions, one has

$$
i_{Y}=\min (r+s, 1+a-r d) \text { and } i_{X}=\min (r-1, s+1,1+a-d),
$$


which leads to many examples satisfying $i_{X}>i_{Y} \geq 2$. The example of lowest dimension (namely 10) for such $X$ and $Y$ is given when $(a, d, r, s)=(5,1,4,2)$. Let us also say that many of these examples lead to $X$ and $Y$ satisfying $r_{X}<i_{X}$ and $r_{Y}<i_{Y}$. In the case $s=1$, the Fano manifolds $Y$ 's have been considered by Debarre (see Deb01, $\S 5.11)$ to construct Fano manifolds of high degree $\left(-K_{Y}\right)^{\operatorname{dim}(Y)}$.

3.3. Minimal degree of free rational curves. When studying Fano manifolds, one often uses free rational curves, which means rational curves $f: \mathbb{P}^{1} \rightarrow X$ such that

$$
f^{*} T_{X} \simeq \mathcal{O}_{\mathbb{P}^{1}}\left(a_{1}\right) \oplus \cdots \oplus \mathcal{O}_{\mathbb{P}^{1}}\left(a_{\operatorname{dim} X}\right)
$$

with all the $a_{i}$ 's greater or equal to 0 (see Deb01, Chapter 4 for details). One may then introduce another invariant:

$$
f_{X}:=\min \left\{-K_{X} \cdot C \mid C \text { is a free rational curve of } X\right\},
$$

which is of great importance in Hwang and Mok's recent works. It can be interpreted as the minimal anti-canonical degree of rational curves whose deformations cover an open dense subset of $X$. It is an easy exercise to show that if $f: X \rightarrow Y$ is a surjective morphism between Fano manifolds $X$ and $Y$, then $f_{X} \leq f_{Y}$. Of course, in any of the examples above where $i_{X}>i_{Y}$, the rational curves in $Y$ of minimal anti-canonical degree are not free curves and their deformations do not cover any dense open subset of $Y$.

3.4. A final remark on a related question. In the above results, the assumption that both $X$ and $Y$ are Fano is essential: when $\pi: X \rightarrow Y$ is a blow-up with smooth connected center $Z$ between complex manifolds $X$ and $Y$, understanding on which conditions $X$ Fano (resp. $Y$ Fano) implies $Y$ Fano (resp. $X$ Fano) is a completely different question, whose study has been initiaded by Wiśniewski in Wi91. In particular, no condition on the dimension of the center is neither necessary nor sufficient (except of course when $Z$ is a point, see BCW02 for a complete classification) to get one of the implications above: the examples in 3.2 in the particular case where $r d>a \geq d$, give examples of smooth blow-up $\pi: X \rightarrow Y$ between complex manifolds $X$ and $Y$ with $X$ being Fano and $Y$ not.

Thanks to Cinzia Casagrande and Olivier Debarre for their comments on a preliminary version of this Note.

\section{REFERENCES}

[An85] T. Ando. On extremal rays of the higher dimensional varieties. Invent. Math. 81, 347-357 (1985).

[BCW02] L. Bonavero, F. Campana et J.A. Wiśniewski. Variétés complexes dont l'éclatée en un point est de Fano. C. R. Math. Acad. Sci. Paris 334, no. 6, 463-468 (2002).

[BCDD03] L. Bonavero, C. Casagrande, O. Debarre and S. Druel. Sur une conjecture de Mukai. Comment. Math. Helv. 78, 601-626 (2003).

[CMS00] K. Cho, Y. Miyaoka, N. Shepherd-Barron. Characterizations of projective space and applications to complex symplectic manifolds. Higher dimensional birational geometry (Kyoto, 1997), Adv. Stud. Pure Math., 35, Math. Soc. Japan, Tokyo, 1-88 (2002).

[Deb01] O. Debarre. Higher-Dimensional Algebraic Geometry. Universitext, Springer Verlag, (2001).

[IP99] V.A. Iskovskikh, Yu.G. Prokhorov. Fano varieties. Algebraic geometry, V. Encyclopaedia Math. Sci. 47, Springer-Verlag, Berlin, 1999.

[Ko96] J. Kollár. Rational curves on algebraic varieties. Ergebnisse der Mathematik und ihre Grenzgebiete. 3 Folge 032, Springer-Verlag, 1996. 
[La83] R. Lazarsfeld. Some applications of the theory of positive vector bundles. Complete intersections, Lect. 1st Sess. C.I.M.E., Acireale/Italy 1983, Lect. Notes Math. 1092, 29-61 (1984).

[OSS81] C. Okonek, M. Schneider, H. Spindler. Vector bundles on complex projective spaces. Progress in Mathematics, 3. Birkhäuser, Boston, Mass., 1980. vii+389 pp.

[SW90] M. Szurek, J. Wiśniewski. Fano bundles over $\mathbb{P}^{3}$ and $Q^{3}$. Pac. J. Math. 141, No.1, 197-208 (1990).

[Wi91] J. Wiśniewski. On contractions of extremal rays of Fano manifolds. J. Reine Angew. Math. 417, 141-157 (1991).

Laurent Bonavero. Institut Fourier, UMR 5582, Université de Grenoble 1, BP 74. 38402 Saint Martin d'Hères. FRANCE

e-mail : bonavero@ujf-grenoble.fr 